\title{
Personal identity and neuroticism among late adolescents
}

\author{
Indumathy $\mathbf{J}^{1, *}$, Divvyalakshmi N. N. ${ }^{2}$, Ashwini U R ${ }^{3}$, Ashwini K. ${ }^{4}$ \\ 1,2M.Phil Scholar, Dept. of Psychology, Madras School of Social Work, Chennai, Tamil Nadu, ${ }^{3}$ Assistant Professor, Chevalier T \\ Thomas Elizabeth College for Women, Chennai, Tamil Nadu, ${ }^{4}$ Counsellor, Kendriya Vidyalaya Nagercoil, Tamil Nadu, India
}

Corresponding Author:

Email: indumathyjayaprakash@gmail.com

\begin{abstract}
Every human thrives for an identity for themselves; they like to be known to others. The characteristics, beliefs, values, behaviours and thoughts all together form the identity of an individual. In other words it can be said as the concept that is developed about oneself. Neuroticism is one of the big five personality factor which basically the emotional instability of a person. The feelings of anxiety, rumination, fear and frustration is high in individuals who are high in neuroticism. The researchers of this study made an attempt to investigate the connection between personal identity and neuroticism of a person. This area is less studied and thus the study follows the exploratory research design to gain new insights and understandings of the association. The exploration included 127 late adolescents who fall under the age ranging from 18 - 19 years. The participants were selected from 4 different educational institutions, using systematic sampling technique. Aspects of Identity Questionnaire by Cheek, J. M. \& Briggs, S. R. (2013) and Eysenck's personality Inventory, Short Form (1985) were administered to the participants. A statistical analysis of the data is done using parametric tests (Pearson Product Moment Correlation and Independent Sample t test). The results of the data showed a significant negative relationship between personal identity and neuroticism. And also there did not exist any gender difference among the variables of the study. The implication, limitations and suggestions of the study are discussed in the paper.
\end{abstract}

Keywords: Personal Identity, Neuroticism, Late Adolescents.

\section{Introduction Personal identity}

Personal identity is the concept that develops about oneself that evolves over the course of life. This may include aspects of life that one have no control over, such as where one grew up or the colour of skin, as well as choices one make in life, such as how spending time and what one believe. A person demonstrates portions of personal identity outwardly through what one wear and how he or she interacts with other people. An individual may also keep some elements of personal identity to oneself, even when these parts are very important. Personal identity is discussed under the protean term self. And 'self' does sometimes mean 'person'. But it often means something different: some sort of immaterial subject of consciousness, for instance (as in the phrase 'the myth of the self').

Personal identity is contingent and changeable: different factors could have belonged to the way one defines oneself as a person, and what factors those are can change through the years. It contrasts with ethnic or national identification, which is composed kind of the ethnic organization or nation one takes oneself to belong to and the significance one attaches to this.

The problem of this kind of evaluation of personal identity offers sufficient and necessary situations for the identity of the person over time. In the modern philosophy of mind, this concept of personal identity is denoted as the diachronic problem of personal identity. The synchronic problem is grounded within the query of what features or trends characterise a given person at one time.

\section{Neuroticism}

Neuroticism is defined as disturbing thoughts and worrying feelings that are disproportionate to the actual situation of a person's life. Like most personality traits, levels of neuroticism cover a wide range. In small doses, it is harmless, and may actually serve to keep life in balance. However, in extreme cases, neuroticism can turn into a clinical diagnosis of psychoneurosis, defined as "a functional disorder in which feelings of anxiety, obsessional thoughts, compulsive acts, and physical complaints without objective evidence of disease, in various degrees and patterns, dominate the personality.

Neuroticism is a tendency to exist in a negative emotional state. They respond poorly and negatively to stressors. Those who score high on the neuroticism, will likely to blow things out of proportion, more so than other people. They see the bad factors more easily. The silver lining is far and few.

However, it doesn't suggest that they are not in touch with reality. They may not be delusional, just easily distressed and overwhelmed by life's challenges because of their low emotional stability. Neuroticism, which is the state of being neurotic, is NOT the same as Neurosis, which is a personality disorder. .

\section{The 6 Facets of Neuroticism}

In Big Five dimension, Neuroticism, there are 6 facets which further specify the traits that make a person neurotic. These facets will help identify people who are prone to psychological distress.

1. Anxiety: level of Anxiety. How often and effortlessly one feels anxious. 
2. Angry Hostility: tendency to feel anger, frustration or bitterness.

3. Depression: tendency to feel guilt, loneliness, depression and despondency.

4. Self-Consciousness: experience Social Anxiety very frequently

5. Impulsiveness: tendency to give in to cravings and ability to delay gratification.

6. Vulnerability: ability to handle stress

\section{Late adolescence}

Late childhood encompasses the latter a part of the teenage years, between the ages of 15 and 19. The important physical modifications will generally take place during this year, although the body is still developing. The mind continues to expand and reorganize itself, and the potential for analytical and reflective notion is greatly improved. Peer-group opinions have a tendency to be vital at the outset; however their hold deceases as adolescents benefit greater clarity and self-belief of their personal identity and evaluations.

During late adolescents, individuals has a common characteristics of risk taking, as individuals experiment with 'adult behaviour' decreases in the course of late adolescence, as the capability to assess risk and make conscious choices develops. Though, cigarette smoking and experimentation with drugs and alcohol are frequently embraced in the earlier risk-taking stage and then carried through into later adolescence and past into adulthood. For example, it's far predicted that 1 in 5 adolescents aged 13-15 smokes, and around half of those who begin smoking in adolescents continue to achieve this for at least 15 years. The flip side of the explosive brain development that occurs at some point of adolescence is that it can be seriously and completely impaired by using the excessive use of drugs and alcohol.

Late adolescence, girls have a tendency to be at more risker than boys of negative health effects, such as depression, and those risks are frequently magnified by using gender- based discrimination and abuse. Girls are especially vulnerable to eating disorders which include anorexia and bulimia; this vulnerability derives in component from profound anxieties over body image this is fuelled via cultural and media stereotypes of female beauty.

These risks however, late adolescence is a time of possibility, idealism and promise. It's far in these years that adolescents make their way into the world of work or education, determine their own identification and world view and start to interact actively in shaping the world around them.

\section{Need for Study}

Late adolescents is a time of life when very little is normative. It is a stage of common change and exploration that covers many facets of their life such as home, family, work, school, resources, and role. Adolescents tend to take longer to accomplish financial and psychological autonomy and early adulthood experiences vary greatly by gender, race and ethnicity, and social class. They develop a sense of self as an individual and as a person connected to valuable people, groups and peer relations. Neuroticism is one of the reasons which is often debated as a risk factor for psychopathology. Neuroticism decreases over the course of life, but not uniformly across individuals. However, the implications of neuroticism on mental health have not been well studied so far. The aim of this study is to examine the associations between the personality trait of neuroticism and personal identity in late adolescence.

\section{Review of literature}

Adolescent cigarette smoking is increasing health risk behaviour in many societies. The reasons for adolescent smoking are complex; individuals take up this habit as to manage the stress during the difficult and challenging time of adolescence. This study reports data which suggested that adolescent stress, broadly defined, does indeed relate to the decision to commence smoking by young people. However, though the personality characteristic of neuroticism is independently related with this decision, it does not mediate the association between stress and smoking onset. (Byrne, Byrne and Reinhart 1995)

The study examined the relationship between neurotic personality characteristics and sleep habits/problems. The high neuroticism group went to bed later and slept less than did the low neuroticism group. Subjects in the high neuroticism group had significant risk of feelings of sleep insufficiency, tiredness, moodiness, and difficulty waking up in the morning; they also had more daytime sleepiness than did those in the low neuroticism group. The high neuroticism group also suffered from more sleeprelated problems than did the low neuroticism group.

\section{(Gau 2000)}

This study examined the relationship among social self-efficacy, temperament, social competence, and life satisfaction in a sample of 160 early adolescents. Results indicated that social self-efficacy mediated the relationship between extraversion and life satisfaction, but not between neuroticism and life satisfaction. Teacher-rated social competence did not have effects of temperament on life satisfaction. (Fogle, Huebner and Laughlin 2002)

The aim of the research was to examine the associations between the personality trait of neuroticism in adolescence and later psychotic symptoms. Data was collected on neurotic individual at age 14; and psychotic symptoms predominantly subclinical, assessed on the Symptom Checklist (SCL-90), at ages 18 and 21 and a range of potential confounding factors including measures of childhood adversity and co- 
morbid mental disorders. (Goodwin, Fergusson and Horwood 2003)

This study addresses the question whether the link between dissatisfaction with the relationship and neuroticism appears during the partner relationship, or whether it is present before the person enters the relationship. Results showed that a lower degree of satisfaction in partner relationships in mid-life was linked with higher concurrent neuroticism scores. Opposing most of the previous studies, this association appeared for both men and women. Higher neuroticism in adolescence was, however, not significantly associated with satisfaction in partner relationships in mid-life. (Möller 2004)

The study examined whether higher levels of neuroticism in adolescence were associated with poorer cognitive function in midlife. Higher neuroticism at age 13 was associated with poorer performance on tests of verbal ability, verbal fluency, and verbal memory at age 53 in sex-adjusted analyses. Moreover, higher neuroticism was also related with poorer cognitive performance at age 8. After adjustment for childhood cognition or educational attainment, the associations between neuroticism at age 13 and midlife cognition ceased to be statistically significant. (Gale, Deary, Kuh, Huppert and Richard 2010)

The study revealed the associations of Big Five personality traits with dimensions of identity formation (i.e., identification with commitment and exploration in depth) in the domain of education. Study revealed that changes in Big Five personality traits were related to changes in identification with commitment and exploration in depth. Research uncovered that, except for Openness, all Big Five traits predicted educational identity dimensions. Educational identity dimensions only predicted Neuroticism. (Klimstra, Luyckx, Germeijs, Meeus and Goossens 2012)

The aims of the study were to analyse links between adolescent identity development and relationships with peers identified from a literature review, summarize the results, and discuss the theoretical factors that may predict these relationships. Analysis of findings from the systematic literature review revealed that a good relationship with peers is positively related to adolescent identity development (Ragelienė 2016).

\section{Method of investigation Objectives}

1. To assess the Personal Identity of adolescents

2. To assess the Neuroticism of adolescents

3. To find the relationship between Personal Identity and Neuroticism

4. To fine the gender differences in Personal Identity and Neuroticism

\section{Hypotheses}

1. There is a no significant relationship between Personal Identity and Neuroticism
2. There is no significant gender difference in Personal Identity

3. There is no significant gender difference in Neuroticism

\section{Research and Sampling Design}

There are limited reviews that study the variables of the current study. Hence the study follows the exploratory research design to get new insights about the relationship between Personal Identity and Neuroticism.

The samples of the study are 127 late adolescents whose age range from $18-19$ years. The participants are selected from 4 different academic institutions, selected using lottery method of simple random sampling technique.

\section{Tools}

Aspects of Identity Questionnaire (AIQ-IV) by Cheek, J. M. \& Briggs, S. R. (2013).

This questionnaire consists of 45 statements with 5 alternatives namely " $1=$ Not important to my sense of who I am; 2 = Slightly important to my sense of who I am; 3 = Somewhat important to my sense of who I am; 4 = Very important to my sense of who I am; 5 = Extremely important to my sense of who I am". The questionnaire is divided into 4 dimensions namely Personal Identity Orientation, Relational Identity Orientation, Social Identity Orientation and Collective Identity Orientation. From this the items under Personal Identity are alone chosen for the study (i.e items 258 11141821252732 ). The sum of these 10 items is taken as the score for personal identity. The Cronbach's Coefficient alpha is a preferred statistic used to indicate the level of internal consistency which is 0.73 . The tool also has good content validity.

Eysenck's Personality Questionnaire - Eysenck, H. J., \& Eysenck, S. B. G. (1985)

It is a questionnaire to assess the personality traits of a person, which consists of 48 items. This scale measures three dimensions namely introversion, psychoticism and Neuroticism. The 12 items of neuroticism was derived to measure. The answer of yes to the following items are scored one each $1,5,9,13$, $17,21,25,30,34,38,42,46$. The test-retest reliability of the extraversion, neuroticism, psychoticism and lie scales was $0.84,0.82,0.69$, and 0.69 , respectively. Kuder-Richardson alpha coefficients of the scales for extraversion, neuroticism, psychoticism, and lie were $0.78, \quad 0.65,0.42$, and 0.64 , respectively. The questionnaire also has a good construct and concurrent validity.

Statistical Analysis

The scores of the sample were statistically analysed using parametric test in SPSS 20 version. 
1. Karl Pearson Product Moment Correlation Relationship between Personal Identity and Neuroticism

2. Independent Sample t test - Gender differences in Personal Identity and Neuroticism

\section{Results and discussion}

Table 1: Correlation between Personal Identity and Neuroticism

\begin{tabular}{|c|c|c|}
\hline & Variables & $\mathbf{r}$ \\
\hline & Personal Identity & \multirow{2}{*}{$-0.373 * *$} \\
\hline & Neuroticism & \\
\hline
\end{tabular}

The product moment correlation indicated that personal identity and neuroticism was negatively correlated. The relationship was significant at 0.01 level. In other words the variables are interdependent on each other.

When the personal identity is low the level of neuroticism is high and when the neuroticism level is low the personal identity is high. One variable increases when the othe variable decreases.

Thus the hypothesis stating "There is a no significant relationship between Personal Identity and Neuroticism" is rejected.

Table 2: Mean scores and the level of significance of the variables on the basis of gender

\begin{tabular}{|l|c|c|c|c|c|}
\hline \multicolumn{1}{|c|}{ Variables } & Gender & $\mathbf{N}$ & Mean & SD & t value \\
\hline $\begin{array}{l}\text { Personal } \\
\text { Identity }\end{array}$ & Female & 64 & 40.97 & 5.303 & \multirow{2}{*}{$1.198^{\mathrm{NS}}$} \\
\cline { 2 - 5 } & Male & 63 & 42.16 & 5.884 & \\
\hline \multirow{2}{*}{ Neuroticism } & Female & 64 & 2.52 & 1.574 & \multirow{2}{*}{$1.031^{\mathrm{NS}}$} \\
\cline { 2 - 5 } & Male & 63 & 2.38 & 1.717 & \\
\hline
\end{tabular}

NS - Not Significant

The statistical analysis of the data using independent sample $t$ test for gender differences shows that there is no significant differences among the variables. In other words girls and boys did not differ significantly in Personal Identity and Neuroticism.

Thus the hypotheses stating "There is no significant gender difference in Personal Identity" and "There is no significant gender difference in Neuroticism" are accepted.

\section{Significance of the study}

The current study highlighted the need for identity for an individual. When an individual struggles to find an identity for oneself, they face a lot of problems and confusions. Adolescent stage is the period where the individual strives hard to create an identity or stand for themselves. During this period the role of parents, teachers and peers have a vital role for identity formation. They are the primary sources from whom a person learns and imitates to grow. Thus the support and guidance of these people and also the environment is very important to help an individual to grow in a healthy and efficient manner.

\section{Limitations and Recommendations}

1. The sample size is very small for generalization. Large sample size and representation from different places of the state would be more reliable.

2. Workshops and training programs for adolescents may help them to develop themselves in an efficient manner.

3. Interventional study, using workshops and programs can be done.

\section{References}

1. Catharine R. Gale, Ian J. Deary, Diana Kuh Felicia and Huppert Marcus Richards Neuroticism in Adolescence and Cognitive Function in Midlife in the British 1946 Birth Cohort. The Journals of Gerontology: Series B, Volume 65B, Issue 1, 1 January 2010, Pages 5056,https://doi.org/10.1093/geronb/gbp082

2. Goodwin RD, Fergusson DM, Horwood LJ. (2003) Neuroticism in adolescence and psychotic symptoms in adulthood. Psychol Med. Aug;33(6):1089-97, https://www.ncbi.nlm.nih.gov/pubmed/12946093

3. Gau SF (2000). Neuroticism and sleep-related problems in adolescence, Sleep 01 Jun 2000, 23(4):495-502 http://europepmc.org/abstract/med/10875556

4. Möller, K. (2004), The longitudinal and concurrent role of neuroticism for partner relationships. Scandinavian Journal of Psychology, 45: 79-83. doi:10.1111/j.14679450.2004.00381.x

5. D.G. Byrne A.E. Byrne and M.I. Reinhart January (1995). Personality, stress and the decision to commence cigarette smoking in adolescence. Journal of Psychological Research, 39, (1); 53-62 : https://doi.org/10.1016/0022-3999(94)00074-F

6. Livy M. Fogle, E. Scott Huebner, James E Laughlin (2002). The Relationship between Temperament and Life Satisfaction in Early Adolescence: Cognitive and Behavioral Mediation Models. Journal of Happiness Studies, 2002, 3(4); 373 doi:10.1023/A:1021883830847

7. Klimstra TA, Luyckx K, Germeijs V, Meeus WH, Goossens L. (2012) Personality traits and educational identity formation in late adolescents: longitudinal associations and academic progress. J Youth Adolesc. Mar;41(3):346-61. doi: 10.1007/s10964-0119734-7.

8. Tija Ragelienè and J Can Acad. Links of Adolescents Identity Development and Relationship with Peers: A Systematic Literature Review. Child Adolesc Psychiatry. 2016; 25(2): 97-105.

https://www.ncbi.nlm.nih.gov/pmc/articles/PMC4879949 